\title{
COVID-19 na mulher
}

\author{
Sofia Andrade de Oliveira
}

1 Fez Residência Médica em Ginecologia e Mestrado na Universidade Federal de São Paulo (Unifesp), especialização em Reprodução Humana na Universidade Rey Juan Carlos em Madrid e é professora de Ginecologia da Universidade do Estado da Bahia (UNEB).

OLIVEIRA, S. A. COVID-19 na mulher. In: BARRAL-NETTO, M.; BARRETO, M. L.; PINTO JUNIOR, E. P.; ARAGÃO, E. (org.). Construção de conhecimento no curso da pandemia de COVID-19: aspectos biomédicos, clínico-assistenciais, epidemiológicos e sociais. Salvador: Edufba, 2020. v. 1. DOI: https://doi.org/10.9771/9786556300443.007. 


\section{Vantagens do sexo feminino na infecção pelo SARS-CoV-2}

Em muitos países, a incidência de óbitos por COVID-19 em homens equivale a mais que o dobro de casos de óbitos em mulheres. Dados de estudos epidemiológicos anteriores corroboram com as observações emergentes atuais, que indicam uma relação importante entre o sexo e a infecção pelo coronavírus e sua evolução clínica. (GEDHARD et al., 2020) Cerca de $60 \%$ das mortes por COVID-19 são reportadas em homens. (GEMMATI et al., 2020)

As diferenças entre os sexos quanto à intensidade, à prevalência, à gravidade e à mortalidade nas infecções já foram descritas em outras patologias virais. A tendência na mulher é sempre evidenciar uma melhor resposta às infecções tanto a nível imunológico humoral quanto celular. Esse fenômeno pode estar relacionado às diferenças nos níveis hormonais estrogênicos e androgênicos entre os sexos, já que estrogênio e testosterona se ligam a receptores de células de atividade imunológica. Sabe-se que o estrogênio está envolvido, quando em altos níveis, com a supressão da resposta imune inata e quando em baixos níveis, relaciona-se com a atividade imunoestimulante. A ADAM metalopeptidase (ADAM17) associada à atividade da Enzima Conversora de Angiotensina 2 (ACE2), 
possui sua expressão regulada pelos níveis de estrogênio. (CONTI; YOUNES, 2020)

Porém, essas diferenças podem também estar relacionadas à expressão não balanceada de genes nos cromossomos $\mathrm{X}$ e Y já que muitos genes relacionados à atividade imunológica estão no cromossomo X e parecem estar mais ativados nas células imunes da mulher. (GEDHARD et al., 2020; GEMMATI et al., 2020)

$\mathrm{A}$ atividade gênica produzida por um único cromossomo $\mathrm{X}$ é satisfatória para o ser humano. O fato de ter dois cromossomos $\mathrm{X}$ diferentes - um de origem paterna e um materno - faz com que a mulher inative um dos cromossomos X em cada célula. Desse modo, a mulher tem a possibilidade de ter dois tipos celulares diferentes em todos os tecidos, fenômeno também conhecido como mosaicismo. Esse fato gera um ambiente de melhor adaptabilidade e plasticidade na mulher, principalmente nas mais jovens quando a ativação ou inativação de alelos no cromossomo $\mathrm{X}$ acontece de modo mais eficaz. $\mathrm{O}$ tabagismo parece também influenciar de forma negativa nesse controle de ativação gênica. Desse modo, quando existe alguma mutação heterozigótica que possa interferir negativamente no equilíbrio imunológico, a célula acaba por ativar o alelo não mutado. Assim, através do mosaicismo celular, as mulheres modulam melhor a inflamação. (GEDHARD et al., 2020; GEMMATI et al., 2020; CONTI; YOUNES, 2020)

Um estudo de coorte, com 93 pacientes diagnosticados com COVID-19 admitidos em um hospital, comparou a carga viral, identificada através do swab nasofaringeo, e os níveis de Imunoglobulina G (IgG) nos homens e nas mulheres e não evidenciou diferença estatisticamente significativa. No entanto, quando foram avaliados os níveis de 71 citocinas pró-inflamatórias e quimiocinas, os níveis de Fatores de Necrose Tumoral (TNF) foram significativamente maiores nas mulheres, assim como a ativação das células T CD8+. Os homens tiveram níveis mais elevados de Ligante 5 de 
quimiocina CC (CCL5), Interleucina 8 (IL-8) e Ligante 10 de quimiocina com motivo C-X-C (CXCL10). Quando avaliados os desfechos, a resposta mais pobre das células T CD8+ e a elevação dos níveis de IL-8, observadas nos homens, foram associadas a um pior prognóstico. (TAKAHASHI et al., 2020)

Uma revisão sistemática sobre transcriptômica, com dados genômicos, identificou que as alterações gênicas nos receptores ACE2 se expressam no homem com maior ênfase do que na mulher e, além disso, com atividade aumentada pelo controle dos hormônios sexuais masculinos. (TUKIAINEN et al., 2017) Além disso, o receptor ACE2 da mulher parece ter uma afinidade diferente para a proteína spike do SARS-CoV-2. (CONTI; YOUNES, 2020)

Sabe-se que predisposições genéticas envolvidas no desbalanceamento ACE1/ACE 2, principalmente quando associado à idade avançada e a condições de risco cardiovascular adquiridas, podem levar a disfunções endoteliais, patologias vasculares e apoptose de células alveolares, acelerando o dano pulmonar e piorando a condição infecciosa pelo coronavírus. Esse desbalanceamento parece estar mais presente em homens que em mulheres e um dos fatores levantados é o fato do gene que regula a atividade da ACE2 estar presente no cromossomo X. Desse modo, mutações ou polimorfismos que levem a uma expressão maior de ACE1 podem se expressar de modo mais evidente nos homens do que nas mulheres já que não existe, no homem, uma capacidade de compensar a mutação com a presença de outro alelo não mutado, como já relatado acima no caso das mulheres. (GEMMATI et al., 2020)

Em uma situação de infecção por SARS-CoV-2, a atividade da ACE2 já está suprimida pela ligação ao vírus, pela atividade da ADAM17 ou pelo desequilíbrio ACE1/ACE2 e, quando coexiste com alguma mutação que induza a uma maior expressão de ACE1, o dano tecidual pelo vírus pode acontecer de modo mais acentuado. Quando presente uma mutação que favoreça ainda mais 
essa supressão, o risco de complicações aumenta. (GEMMATI et al., 2020)

\section{ACE2 no útero e na vagina}

A angiotensina II também tem seu papel a nível uterino na regeneração endometrial, na atividade miometrial e na indução da menstruação através da vasoconstricção das artérias espiraladas. (JING et al., 2020) O RNAm ACE2 foi identificado no útero de humanos - mais nas células epiteliais do que nas estromais e, mais acentuadamente, na fase secretiva do que na proliferativa - e também na vagina. É importante destacar que, em estudos experimentais com camundongos infectados pelo SARS-CoV-2, foi evidenciada uma alta taxa de infecção por via sexual, sugerindo a possibilidade de transmissão sexual do SARS-CoV-2 em humanos. (AZAMORA et al., 2020; JING et al., 2020)

Um relato de caso italiano evidenciou através da coleta por swab de secreção vaginal, um quantitativo de Proteína C-Reativa (PCR) positivo para SARS-CoV-2 em uma senhora de 65 anos com diagnóstico de COVID-19, aventando a possibilidade de transmissão da infecção por via sexual. (SCORZOLINI et al., 2020)

\section{ACE2 no ovário}

A angiotensina II atua em diversos mecanismo ovarianos, desde a indução da secreção de hormônios - estrogênio e progesterona - ao desenvolvimento folicular, maturação e atresia dos oócitos, indução da ovulação e manutenção do corpo lúteo. Desse modo, a expressão do ACE2 foi relatada em grande quantidade nas células da granulosa e no estroma ovariano (GENECARDS, 2020; 
PAN et al., 2013) e, assim, os ovários e os óvulos seriam alvo em potencial do SARS-CoV-2 (ZOU et al., 2020), porém, até o momento, não temos dados suficientes para confirmar essa hipótese nem para avaliar as consequências de uma provável presença do vírus no ovário.

Até o momento, não temos informação se o SARS-CoV-2 utiliza receptores ACE2 nos ovários ou no útero e não possuímos dados sobre o impacto da infecção nos oócitos ou no desenvolvimento embrionário.

Sabe-se também que a infecção por SARS-CoV-2 pode afetar os tecidos do sistema reprodutor masculino gerando orquite grave. A orquite, quando associada a febre, pode reduzir a concentração espermática e motilidade, gerando impacto na fertilidade. (CARLSEN et al., 2003)

\section{Gestação e SARS-GoV-2}

Durante a gestação, as alterações hormonais levam a mudanças fisiológicas no sistema imune feminino, que induzem a um estado de imunotolerância e favorecem, assim, a permanência do feto. Esse evento ocorre através da atenuação fisiológica da resposta de linfócitos T Helper 1 (Th1), com consequente redução dos níveis de citocinas pró-inflamatórias - IFNg, IL-1b, IL-6 e IL-12 - e dominância da resposta Th2, com a expressão de citocinas anti-inflamatórias - IL-4 e IL-10. Tais mudanças, apesar de favorecerem a evolução da gestação, acabam por aumentar a suscetibilidade materna a infecções por patógenos intracelulares como o SARS-CoV-2. (WONG et al., 2004)

Ao mesmo tempo, muitas são as mudanças fisiológicas no sistema cardiorrespiratório da gestante: 
1. dilatação dos capilares da árvore brônquica, gerando edema e dificultando a respiração nasal;

2. respiração de predominância diafragmática;

3. elevação do diafragma por compressão mecânica do volume uterino aumentado no terceiro trimestre;

4. maior consumo de oxigênio pelo binômio mãe-bebê. (RACICOT; MOR, 2017)

Essas modificações não só geram susceptibilidade às infecções respiratórias, como também retardam o diagnóstico e favorecem a progressão de uma doença focal para uma consolidação difusa pulmonar bilateralmente. (DASHRAATH et al., 2020; GOTTFREDSSON, 2008)

Apesar desse risco teórico, algumas publicações, com dados epidemiológicos, mostram que grávidas vêm apresentando um risco de contaminação e quadro clínico, na COVID-19, semelhante às mulheres não grávidas de mesma idade (FAVRE et al., 2020; FERNÁNDEZ-CARRASCO et al., 2020; LIANG; ACHARYA, 2020): a maioria evolui de modo assintomático ou apresenta sintomas leves a moderados como tosse, febre, dispneia leve, cefaleia, sintomas gastrointestinais ou anosmia. (FAVRE et al., 2020) Um estudo observacional com 155 gestantes assintomáticas, que foram admitidas para parto cesariano eletivo, evidenciou que 15,5\% estavam infectadas por SARS-CoV-2. (BIANCO et al., 2020)

Por outro lado, alguns estudos têm mostrado um risco aumentado de complicações gestacionais como abortamento, pré-eclâmpsia, restrição do crescimento intrauterino, parto cesariano, parto prematuro, sofrimento fetal e rotura prematura de membranas, principalmente quando a gestante se encontra internada. (CHEN, H. et al., 2020; FERNÁNDEZ-CARRASCO et al., 2020) Uma meta-análise mostrou um aumento do risco $(2,4 \%)$ de óbito 
neonatal precoce e de internação em Unidade de Terapia Intensiva (UTI) neonatal. (DI MASCIO et al., 2020)

Outro fator que pode predispor a complicações gestacionais é o status tromboinflamatório que a COVID-19 impõe. Através da reação inflamatória com a "tempestade de citocinas" e, também, através de uma possível disfunção hepática, a paciente infectada pode apresentar um estado de hipercoagulabilidade. No caso da gestação e no puerpério, o estado fisiológico já instalado de hipercoagulabilidade aumenta ainda mais o risco de eventos tromboembólicos durante a infecção por SARS-CoV-2. (LEFFERT et al., 2018)

\section{Transmissão vertical}

A transmissão vertical é um evento possível do ponto de vista teórico, já que a ACE2 é ricamente expressa na placenta, nas células da decídua, no cordão umbilical e no âmnio. Na placenta, a expressão da ACE2 é ainda mais enfática na gestação inicial entre seis e nove semanas. (PRINGLE et al., 2011) De acordo com o GeneCards (2020), a expressão da ACE2 é maior na placenta do que nos pulmões.

Uma série de casos em Nova Iorque com 32 parturientes com COVID-19, estudou a placenta de 11 parturientes e evidenciou três placentas com PCR positivo para SARS-CoV-2. Nenhum dos 11 recém-nascidos apresentou PCR positivo. (PENFIELD et al., 2020)

Um outro relato de caso relevante, ainda sob revisão, diagnosticou a presença molecular da infecção pelo SARS-CoV-2 (genes E e S) na placenta. Além da placenta, também foi evidenciado PCR positivo (genes E e $S$ ) através de coleta durante a cesárea, no líquido amniótico, no sangue e nas secreções retal e nasofaríngea do recém-nascido. A Figura 1 ilustra o exame histopatológico da placenta do caso descrito, com a presença de zonas de 
infarto e deposição de fibrina perivilositária, descritos no relato. (VIVANTI et al., 2020)

Figura 1 - Estudo anatomopatológico da placenta de paciente com COVID-19

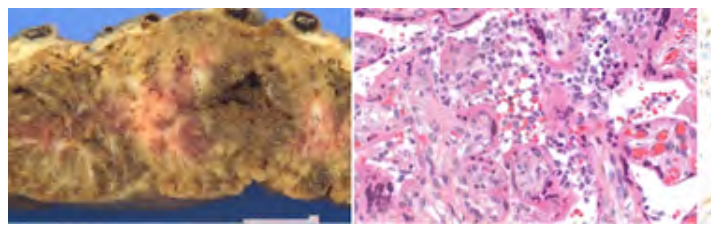

Fonte: Vivanti e demais autores (2020, p. 10).

Mais um relato de três casos em parturientes no terceiro trimestre de gestação com COVID-19 também evidenciou vários graus de depósito de fibrina e presença de infarto placentário, mas em todos os três casos as placentas foram negativas em estudo molecular para SARS-CoV-2. (LAMOUROUX et al., 2020)

Recentemente, a infecção COVID-19 foi identificada em recém-nascidos de dois e quatro dias de vida, através de swab nasofaríngeo e swab anal. Outro neonato de mãe com COVID-19 teve elevada titulação de anticorpos Imunoglobulina M (IgM) com apenas 2 horas após o nascimento. (DONG et al., 2020) Levando em consideração que anticorpos IgM não conseguem passar pela placenta e que a detectação de anticorpos nas células humanas não são observadas em menos 96 horas de cultura, especula-se a infecção intrauterina pelo COVID-19. (PRINGLE et al., 2011)

Entretanto, outros relatos de caso publicados não identificaram presença do vírus no líquido amniótico. (CHEN, H., et al., 2020; CHEN, Y. et al., 2020; LI et al., 2020; ZHU et al., 2020) Mais uma informação relevante é a não existência, até o momento, de evidência de teratogenicidade do SARS-CoV-2. 


\section{Transmissão pelo leite}

O ACE2 também foi evidenciado nas mamas. Evidências ainda fracas sugerem que o SARS-CoV-2 pode estar presente no leite materno, mas esses estudos não mostram com clareza o método de coleta do leite, o período em que foi feita a coleta nem apresentam a validação do teste PCR-TR para o leite materno.

Um estudo de revisão avaliou 13 publicações sobre leite materno e COVID-19 e das 48 amostras de leite materno - de mães portadoras de COVID-19 - avaliadas no total, uma única amostra de leite apresentou PCR positivo para SARS-CoV-2, sugerindo um risco de transmissão através da amamentação. (WU et al., 2020) Em outra publicação, duas amostras, de uma mesma paciente, apresentaram anticorpo IgG positivo para SARS-CoV-2. (LACKEY et al., 2020)

Uma revisão sistemática, feita com 22 estudos e um total de 83 recém-nascidos de parturientes portadoras de COVID-19, evidenciou um total de nove infecções por SARS-CoV-2 nos recém-nascidos (PCR positivo ou níveis elevados de anticorpos). Dentre os nove casos, seis tiveram avaliação do leite materno e secreção vaginal. Não foi evidenciado PCR positivo em nenhuma amostra. (ZENG et al., 2020)

Dado o exposto, percebe-se que o sexo feminino parece ter uma vantagem durante a infecção pelo SARS-CoV-2, por ter uma plasticidade maior durante a sua resposta imunológica na infecção. Por outro lado, quando gestante, as modificações fisiológicas da gestação parecem desfavorecer o quadro infeccioso e, além disso, a infecção pelo SARS-CoV-2 pode aumentar o risco de complicações gestacionais como abortamento, trabalho de parto prematuro, óbito fetal. Com relação ao risco de transmissão vertical, relatos de caso evidenciam o potencial de transmissão, porém, felizmente, não foram observados casos de teratogenicidade ou complicações neonatais importantes. Pela escassez de dados mais 


\section{robustos, é necessário aguardar maiores evidências científicas para conclusões mais concretas.}

\section{Referências}

ALZAMORA, M. C. et al. Severe COVID-19 during Pregnancy and Possible Vertical Transmission. American Journal of Perinatology, New York, v. 37, n. 8, p. 861-865, Apr. 2020. DOI: 2020. 10.1055/s-0040-1710050. Disponível em: https://pubmed. ncbi.nlm.nih.gov/32305046/. Acesso em: 4 de ago. 2020.

BIANCO, A. et al. Testing of patients and Support Persons for Coronavirus Disease 2019 (COVID-19) Infection Before Scheduled Deliveries. Obstetrics \& Gynecology, Hagerstown, v. 136, n. 2, p. 283-287, 2020. DOI: 10.1097/AOG.0000000000003985. Disponível em: https://journals.Iww.com/greenjournal/fulltext/2020/08000/testing of_patients_and_support_persons_for.10.aspx. Acesso em: 30 jul. 2020.

CARLSEN, E. et al. History of febrile illness and variation in semen quality. Human Reproduction, Oxford, v. 18, n. 10, p. 2089-2092, Oct. 2003. DOI: 10.1093/ humrep/deg412. Disponível em: https://academic.oup.com/humrep/ article/18/10/2089/622725. Acesso em: 30 jul. 2020.

$\mathrm{CHEN}, \mathrm{H}$. et al. Clinical characteristics and intrauterine vertical transmission potential of COVID-19 infection in nine pregnant women: a retrospective review of medical records. The Lancet, London, v. 395, n. 10226, p. 809-815, Mar. 2020.

CHEN, Y. et al. Infants Born to Mothers with a New Coronavirus (COVID-19). Frontiers in Pediatrics, Lausanne, v. 8, n. 104, 2020. DOI: 10.3389/fped.2020.00104. Disponível em: https://www.researchgate.net/publication/339952688_Infants_ Born_to_Mothers_With_a_New_Coronavirus_COVID-19. Acesso em: 30 jul. 2020.

CONTI, P.; YOUNES A. Coronavirus COV-19/SARS-COV-2 Affects Women Less Than Men: Clinical Response To Viral Infection. Journal Of Biological Regulators \& Homeostatic Agents, Birmingham, AL, v. 34, n. 2, p. 339-343, 2020.

DASHRAATH, P. et al. Coronavirus disease 2019 (COVID-19) pandemic and pregnancy. American Journal of Obstetrics \& Gynecology, New York, v. 222, n. 6, p. 521-531, Mar. 2020. DOI: 10.1016/j.ajog.2020.03.021. Disponível em: https://www.sciencedirect.com/science/article/pii/S0002937820303434 . Acesso em: 4 ago. 2020 
DI MASCIO, D. et al. Outcome of Coronavirus spectrum infections (SARS, MERS, COVID 1 -19) during pregnancy: a systematic review and meta-analysis. American Journal of Obstetrics \& Gynecology, New York, v. 2, n. 2, p. 1-9, May 2020. DOI: 10.1016/j.ajogmf.2020.100107. Disponível em: https://www. uptodate.com/contents/zh-Hans/severe-acute-respiratory-syndrome-sars/print. Acesso em: 4 ago. 2020.

DONG, L. et al. Possible Vertical Transmission of SARS-CoV-2 From an Infected Mother to Her Newborn. JAMA, Chicago, v. 323, n. 18, p. 1846-1848, Mar. 2020.

FAVRE, G. et al. Guidelines for pregnant women with suspected SARS-CoV-2 infection. The Lancet: infectious diseases, New York, v. 20, n. 6, p. 652-653, 2020.

FERNÁNDEZ-CARRASCO, F. J. et al. Coronavirus COVID-19 Infection and Breastfeeding: An Exploratory Review. Revista Española de Salud Pública, [Madrid], v. 94, p. 1-9, 2020.

GEDHARD, C. et al. Impact of sex and gender on COVID-19 outcomes in Europe. Biology of Sex Differences, London, v. 11, n. 29, p. 1-13, May 2020. DOI: 10.1186/ s13293-020-00304-9. Disponível em: https://bsd.biomedcentral.com/ articles/10.1186/s13293-020-00304-9. Acesso em: 30 jul. 2020.

GEMMATI, D. et al. COVID-19 and Individual Genetic Susceptibility/Receptivity: Role of ACE1/ACE2 Genes, Immunity, Inflammation and Coagulation. Might the Double X-Chromosome in Females Be Protective against SARS-CoV-2 Compared to the Single X-Chromosome in Males? International Journal of Molecular Sciences, Basel, v. 21, n. 10, p. 3474, May 2020.

GENECARDS. ACE2 Gene (Protein Coding). [S. I.], 2020.

Disponível em: https:// www.genecards.org/cgi-bin/carddisp. pl?gene=ACE2\#protein_expression. Acesso em: 30 jul. 2020.

GOTTFREDSSON, M. The Spanish flu in Iceland 1918. Lessons in medicine and history. Laeknabladid, Reykjavik, v. 94, n. 11, p. 737-745, 2008.

JING Y. et al. Potential influence of COVID-19/ACE2 on the female reproductive system. Molecular Human Reproduction, Oxford, v. 26, n. 6, p. 367-373, 2020. DOI: 10.1093/molehr/gaaa03. Disponível em: https://pubmed. ncbi.nlm.nih.gov/32365180/ Acesso em: 4 ago. 2020.

LACKEY, K. A. et al. SARS-COV-2 and human milk: what is the evidence? MedRxiv, [s. I.], p. 1-26, 2020. DOI: 10.1101/2020.04.07.20056812. Disponível em: https://www.medrxiv.org/content/10.1101/2020.04.07.20056812v2. Acesso em: 4 ago. 2020. 
LAMOUROUX, X. et al. Evidence for and against vertical transmission for SARS-CoV-2 (COVID-19). American Journal of Obstetrics \& Gynecology, New York, v. 223, n. 1, p. 91-94, 2020. DOI: 10.1016/j.ajog.2020.04.039. Disponível em: https:// www.ajog.org/article/S0002-9378(20)30524-X/fulltext. Acesso em: 4 ago. 2020.

LEFFERT, L. et al. Members of the SVTET. The Society for Obstetric Anesthesia and Perinatology Consensus Statement on the Anesthetic Management of Pregnant and Postpartum Women Receiving Thromboprophylaxis or Higher Dose Anti- coagulants. Anesthesia and Analgesia, Baltimore, v. 126, n. 3, p. 928-944, 2018.

LIANG, H.; ACHARYA, G. Novel coronavirus disease (COVID-19) in pregnancy: what clinical recommendations to follow? Acta Obstetricia et Gynecologica Scandinavica, Malden, v. 99, p. 439-442, Mar. 2020.

LI, N. et al. Maternal and neonatal outcomes of pregnant women with COVID-19 pneumonia: a case-control study. Mar. 2020. DOI: 10.1101/2020.03.10.20033605. Disponível em: https://www.medrxiv.org/content/10.1101/2020.03.10.20033605v1. Acesso em: 4 ago. 2020.

PAN, P. P. et al. Angiotensin-converting enzymes play a dominant role in fertility. International Journal of Molecular Sciences, Basel, v. 14, n. 10, p. 21071-21086, Oct. 2013.

PENFIELD, C. A. et al. Detection of SARS-COV-2 in Placental and Fetal Membrane Samples. American Journal of Obstetrics \& Gynecology MFM, Switzerland, 2020. DOI: 10.1016/j.ajogmf.2020.100133. Disponível em: https:// pubmed.ncbi.nlm.nih.gov/32391518/. Acesso em: 30 jul. 2020.

PRINGLE, K. G. et al. The expression. and localization of the human placental prorenin/renin-angiotensin system throughout pregnancy: roles in trophoblast invasion and angiogenesis? Placenta, London, v. 32, n. 12, p. 956-962, Dec. 2011.

RACICOT, K.; MOR, G. Risks associated with viral infections during pregnancy. The Journal of Clinical Investigation, Ann Arbor, v. 127, n. 5, p. 1591-1599, May 2017.

SCORZOLINI, L. et al. Comment of the potential risks of sexual and vertical transmission of COVID-19 infection. Clinical Infectious Diseases, Oxford, Apr. 2020. DOI: 10.1093/cid/ciaa445. Disponível em: https://academic.oup.com/cid/article/ doi/10.1093/cid/ciaa445/5820874. Acesso em: 4 ago. 2020.

TAKAHASHI, T. et al. Sex Differences in immune responses to SARS-COV-2 that underlie disease outcomes. MedRxiv, [s. I.], London, v. 20, n. 8, p. 461, 2020. DOI: 10.1038/s41577-020-0378-2. Disponível em: https://www.medrxiv.org/ content/10.1101/2020.06.06.20123414v2. Acesso em: 30 jul. 2020. 
TUKIAINEN, T. et al. Landscape of $X$ chromosome inactivation across human tissues. Nature, London, v. 550, p. 244-248, 2017.

VIVANTI, A. et al. Transplacental transmission of SARS-COV-2 infection. Maternal \& Fetal Medicine, [s. I.], p. 1- 2020. DOI: 10.21203/rs.3.rs-28884/v1. Disponível em: https://www.researchsquare.com/article/rs-28884/v1. Acesso em: 30 jul. 2020.

WONG, S. F. et al. Pregnancy and perinatal outcomes of women with severe acute respiratory syndrome. General Obstetrics and Gynecology, New York, v. 191, n. 1, p. 292-297, 2004.

WU, Y. et al. Coronavirus disease 2019 among pregnant Chinese women: case series data on the safety of vaginal birth and breastfeeding. BJOG: an International Journal of Obstetrics and Gynaecology, Oxford, p. 1109-1115, 2020.

DOI: 10.1111/1471-0528.16276. Disponível em: https://obgyn.onlinelibrary.wiley. com/doi/abs/10.1111/1471-0528.16276. Acesso em: 30 jul. 2020.

ZENG, H. et al. Antibodies in infants born to mothers with COVID-19 pneumonia. JAMA, Chicago, v. 323, n. 18, p. 1848-1849, 2020.

ZHU, H. et al. Clinical analysis of 10 neonates born to mothers with 2019-nCoV pneumonia. Translational Pediatrics, Hong Kong, v. 9, n. 1, p. 51-60, Feb. 2020. DOI: http://dx.doi.org/10.21037/tp.2020.02.06. Disponível em: https://www.ncbi. nlm.nih.gov/pmc/articles/PMC7036645/. Acesso em: 4 ago. 2020.

ZOU X. et al. Single-cell RNA-seq data analysis on the receptor ACE2 expression reveals the potential risk of different human organs vulnerable to 2019-nCoV infection. Frontiers of Medicine, Hudson, v. 14, p. 185-192, 2020. DOI: 10.1007/ s11684-020-0754-0. Disponível em: https://link.springer.com/content/ pdf/10.1007/s11684-020-0754-0.pdf. Acesso em: 4 ago. 2020. 